\title{
Partner symmetries and non-invariant solutions of four-dimensional heavenly equations
}

\author{
A A Malykh ${ }^{1}$, Y Nutku ${ }^{3}$ and M B Sheftel ${ }^{1,2,3}$
}

\author{
${ }^{1}$ Department of Higher Mathematics, North Western State Technical \\ University, Millionnaya St. 5, 191186, St. Petersburg, Russia \\ 2 Department of Physics, Boğaziçi University, 34342 Bebek, Istanbul, \\ Turkey \\ ${ }^{3}$ Feza Gürsey Institute, PO Box 6, Çengelköy, 81220 Istanbul, Turkey \\ E-mail: specarm@online.ru,nutku@gursey.gov.tr, sheftel@gursey.gov.tr and \\ mikhail.sheftel@boun.edu.tr
}

\begin{abstract}
We extend our method of partner symmetries to the hyperbolic complex Monge-Ampère equation and the second heavenly equation of Plebañski. We show the existence of partner symmetries and derive the relations between them. For certain simple choices of partner symmetries the resulting differential constraints together with the original heavenly equations are transformed to systems of linear equations by an appropriate Legendre transformation. The solutions of these linear equations are generically non-invariant. As a consequence we obtain explicitly new classes of heavenly metrics without Killing vectors.

PACS numbers: 04.20.Jb, 02.40.Ky

Mathematics Subject Classification: 35Q75, 83C15
\end{abstract}

\section{Introduction}

After advances in Twistor Theory it became natural to consider Ricci-flat metrics on 4-dimensional complex manifolds. In his pioneering paper [1] Plebañski introduced his first and second heavenly equations for a single potential governing such metrics. From solutions of these equations we obtain the corresponding heavenly metrics which give solutions of the complex vacuum Einstein equations possessing the property of (anti-)self-duality. The 
problem here is to reduce these solutions to 4-dimensional real metrics with Lorentzian signature. There are two important real cross sections of the complex metrics governed by the first heavenly equation, namely Kähler metrics with Euclidean or ultra-hyperbolic signature. The first heavenly equation in these cases coincides with the elliptic and hyperbolic complex Monge-Ampère equation $(C M A)$ respectively which have applications to important problems in physics and geometry. In particular, for the elliptic case of $C M A$ some solutions can be interpreted as gravitational instantons. The most important gravitational instanton is the Kummer surface $K 3$ [2] The explicit construction of $K 3$ metric is a challenging problem. One of the basic difficulties is that the metric should have no Killing vectors and hence the corresponding solution of $C M A$ should have no symmetries, i.e. be a non-invariant solution.

This was our motivation to study the problem of constructing non-invariant solutions of complex Monge-Ampère equations. In the elliptic case we have recently developed the method of partner symmetries appropriate for this problem and obtained certain classes of non-invariant solutions of $C M A$ and corresponding heavenly metrics with no Killing vectors [3, 4. The starting point of the method was the observation that the determining equation for symmetries of the elliptic $C M A$ can be presented in the form of a total divergence. This allowed us to introduce locally a potential variable, and another key observation was that the potential again satisfied the same determining equation, i.e. also was a symmetry. We called such a pair of original symmetry and its potential partner symmetries. The equations relating partner symmetries are therefore recursion relations mapping any symmetry again into a symmetry of $C M A$ but the corresponding recursion operator is non-local. Thus if we apply it to a local symmetry then a non-local symmetry will be generated. To avoid the explicit use of non-local symmetries, we consider both partner symmetries as local symmetries and the relation between them as invariance condition with respect to a certain resulting non-local symmetry which we never explicitly put into play. Hence the resulting solutions, though invariant with respect to some non-local symmetry, will be non-invariant solutions in the usual sense and hence generate metrics without Killing vectors. This is closely related to the approach of Dunajski and Mason [5] though their 'hidden' symmetries belong to a more special class of non-local symmetries than those constructed from the partner symmetries.

For any particular choice of partner symmetries the relations between them become differential constraints compatible with the original $C M A$ 
equation. We discovered certain useful choices of partner symmetries when $C M A$ together with the differential constraints could be linearized by a Legendre transformation. Solving linear equations and using their solutions in the Legendre transform of the metric we obtained explicitly some classes of Riemannian metrics with Euclidean signature and anti-self-dual curvature that did not admit any Killing vectors. It is worth noting that linearization of particular solution manifolds of PDEs by the Legendre transformation was suggested in 6 .

In the present paper we extend our method of partner symmetries to two more 4-dimensional heavenly equations: hyperbolic complex Monge-Ampère equation $(H C M A)$, which is the other interesting real cross section of the first heavenly equation, and the second heavenly equation of Plebañski. Our method of partner symmetries works because determining equations for symmetries of all these heavenly equations have the structure of total divergence, and the potential for a symmetry is itself a symmetry. Therefore we again have pairs of partner symmetries for all these heavenly equations. We find such choices of partner symmetries when the original heavenly equation together with differential constraints arising from relations between partner symmetries can be linearized by the Legendre transformation. We present solutions of linear equations and the corresponding metrics without Killing vectors.

We study the hyperbolic complex Monge-Ampère equation in the first part of the paper and the second heavenly equation in the second part.

In section 2, for the sake of completeness, we show that if the Kähler potential satisfies $H C M A$ the corresponding Kähler metric has ultra-hyperbolic signature. In section 3 we derive the relations defining partner symmetries from divergence structure of the determining equation for symmetries of $H C M A$ and make our choices of partner symmetries. In section 4 we consider partial Legendre transformation of $H C M A$ and the relations between partner symmetries in the case when both partner symmetries coincide. We also obtain the Legendre transform of the Kähler metric. Making further choices of partner symmetries as translational or dilatational symmetry we arrive at systems of linear PDEs for which we obtain explicitly non-invariant solutions. Using them in the Legendre transformed metric we obtain explicit 4-dimensional metrics without Killing vectors which is justified in section 7

In section 5 we consider the second heavenly equation with the corresponding heavenly metrics. In subsection 5.1 we show the existence of partner symmetries and derive the relations defining them from divergence structure 
of the determining equation for symmetries. In subsection 5.2 we present explicitly basis generators of the total Lie algebra of point symmetries of the second heavenly equation and the table of its commutation relations. The Legendre transform of the second heavenly metric is given in subsection [5.3 In section [6 we consider two simple choices of partner symmetries. In subsection 6.1] we discuss the subcase when both of them coincide with the same translational symmetry. The Legendre transformation of the heavenly equation and differential constraints arising from this choice convert them to a system of linear equations which is easily solved. In subsection 6.2 we choose one of the partner symmetries to be translational symmetry and the characteristic of the other one is set equal to zero. The same Legendre transformation gives us again a linear system which is easily solved. In the generic case all these solutions are non-invariant and the corresponding metrics have no Killing vectors.

In section 7 we analyze the Killing equations for both the Kähler and the second heavenly metrics. We find a first integral of the Killing equations which is a first-order PDE for the metric potential that contains all the information in Killing's equations. We show that for our solutions this existence condition for the Killing vector cannot be satisfied as it implies functional dependence between independent variables.

\section{Hyperbolic complex Monge-Ampère equation and ultra-hyperbolic metrics}

In the famous paper of Plebañski [1] the Einstein vacuum equations in the complex four-dimensional Riemannian space together with the constraint of (anti-)self-duality are reduced to the general complex Monge-Ampère equation $(C M A)$

$$
\left|\begin{array}{ll}
\Omega_{p r} & \Omega_{p s} \\
\Omega_{q r} & \Omega_{q s}
\end{array}\right|=1
$$

governing the metric

$$
d s^{2}=\Omega_{p r} d p d r+\Omega_{p s} d p d s+\Omega_{q r} d q d r+\Omega_{q s} d q d s
$$

where the 'key function' $\Omega$ is a complex-valued function of the complex variables $p, q, r, s$ and we skip the overall constant factor 2 . 
To restrict this general result to physically interesting cases, we require that $\Omega=u$ where $u$ is a real-valued function and the independent variables form two pairs of complex conjugate variables: $p=z^{1}, q=z^{2}, r=\varepsilon \bar{z}^{1}$, $s=\bar{z}^{2}$ with $\varepsilon= \pm 1$. Then the field equation (2.1) takes the form

$$
u_{1 \overline{1}} u_{2 \overline{2}}-u_{1 \overline{2}} u_{2 \overline{1}}=\varepsilon
$$

and the metric (2.2) becomes

$$
d s^{2}=u_{1 \overline{1}} d z^{1} d \bar{z}^{1}+u_{1 \overline{2}} d z^{1} d \bar{z}^{2}+u_{2 \overline{1}} d z^{2} d \bar{z}^{1}+u_{2 \overline{2}} d z^{2} d \bar{z}^{2}
$$

where the subscripts $i, \bar{j}$ denote partial derivatives with respect to $z^{i}, \bar{z}^{j}$ respectively.

To determine the signature of the resulting metric (2.4) and its dependence on $\varepsilon$, we use the tetrad of the Newman-Penrose co-frame $\{l, \bar{l}, m, \bar{m}\}$ [7, 8] corresponding to the metric (2.4)

$$
l=\frac{1}{\sqrt{u_{1 \overline{1}}}}\left(u_{\overline{1} 1} d z^{1}+u_{\overline{1} 2} d z^{2}\right), \quad m=\frac{1}{\sqrt{u_{1 \overline{1}}}} d z^{2}
$$

with $\bar{l}$ and $\bar{m}$ being complex conjugate to $l$ and $m$. Then using the field equation (2.3) in the form

$$
\frac{u_{1 \overline{2}} u_{\overline{1} 2}}{u_{1 \overline{1}}}=u_{2 \overline{2}}-\frac{\varepsilon}{u_{1 \overline{1}}}
$$

we check that the metric

$$
d s^{2}=l \otimes \bar{l}+\varepsilon m \otimes \bar{m}
$$

coincides with (2.4). The form (2.6) implies that the metric (2.4) is Euclidean with the signature $(++++)$ for $\varepsilon=1$ and it is ultra-hyperbolic with the signature $(++--)$ for $\varepsilon=-1$. The difference comes solely from the dependence of the field equation (2.3) on $\varepsilon$ whereas the metric has the same form (2.4) in both cases.

In the first part of this paper we shall be interested in the ultra-hyperbolic metric (2.4) governed by the hyperbolic complex Monge-Ampère equation $(H C M A)$

$$
u_{1 \overline{1}} u_{2 \overline{2}}-u_{1 \overline{2}} u_{2 \overline{1}}=-1
$$

i.e. (2.3) with $\varepsilon=-1$.

The relation between ultra-hyperbolic metrics and $H C M A$ was demonstrated by a different method in [9]. 


\section{Partner symmetries of the hyperbolic complex Monge-Ampère equation}

The determining equation for symmetries of $H C M A$ is the same as for the elliptic $C M A(2.3)$ with $\varepsilon=1[3]$

$$
\square(\varphi)=0, \quad \square=u_{2 \overline{2}} D_{1} D_{\overline{1}}+u_{1 \overline{1}} D_{2} D_{\overline{2}}-u_{2 \overline{1}} D_{1} D_{\overline{2}}-u_{1 \overline{2}} D_{2} D_{\overline{1}}
$$

where $\varphi$ denotes symmetry characteristic [1] and $D_{i}, D_{\bar{i}}$ are operators of total derivatives with respect to $z^{i}, \bar{z}^{i}$ respectively. Our starting point is the divergence form of this equation

$$
D_{1} L_{2} \varphi=D_{2} L_{1} \varphi
$$

where

$$
L_{1}=i\left(u_{1 \overline{2}} D_{\overline{1}}-u_{1 \overline{1}} D_{\overline{2}}\right), \quad L_{2}=i\left(u_{2 \overline{2}} D_{\overline{1}}-u_{2 \overline{1}} D_{\overline{2}}\right) .
$$

Hence there locally exists the potential $\psi$ such that

$$
\psi_{1}=L_{1} \varphi=i\left(u_{1 \overline{2}} \varphi_{\overline{1}}-u_{1 \overline{1}} \varphi_{\overline{2}}\right), \quad \psi_{2}=L_{2} \varphi=i\left(u_{2 \overline{2}} \varphi_{\overline{1}}-u_{2 \overline{1}} \varphi_{\overline{2}}\right) .
$$

Using this definition of $\psi$ and $H C M A$, a straightforward check shows that $\square(\psi)=0$ and thus $\psi$ is also a symmetry characteristic of HCMA together with $\varphi$. Thus equations (3.4) are recursion relations for symmetries of $H C M A$.

We have called such a pair of mutually related symmetry characteristics partner symmetries [3]. Formulas (3.4) can be presented in the form

$$
\psi=R \varphi=D_{1}^{-1} L_{1} \varphi, \quad \psi=R \varphi=D_{2}^{-1} L_{2} \varphi
$$

where $R$ is recursion operator defined on the subspace of partner symmetries.

Solving algebraically the equations complex conjugate to (3.4) with respect to $\varphi_{1}, \varphi_{2}$ and using (2.7) we obtain

$$
\varphi_{1}=L_{1} \psi=i\left(u_{1 \overline{2}} \psi_{\overline{1}}-u_{1 \overline{1}} \psi_{\overline{2}}\right), \quad \varphi_{2}=L_{2} \psi=i\left(u_{2 \overline{2}} \psi_{\overline{1}}-u_{2 \overline{1}} \psi_{\overline{2}}\right)
$$

so that $\varphi$ is expressed through $\psi$ exactly in the same way as $\psi$ is expressed through $\varphi$ in (3.4). This is the basic difference with the elliptic CMA for which we had an extra minus sign: $\varphi_{1}=-L_{1} \psi, \varphi_{2}=-L_{2} \psi$. 
The $H C M A$ itself emerges now as an algebraic consequence of any three equations of the system (3.4), (3.6) on the account of complex conjugate equations.

We will avoid operating explicitly with non-local symmetries and use instead the equations (3.6) only with the point symmetries $\varphi$ and $\psi$. Then these equations are equivalent to an invariance condition for solutions of $H C M A$ with respect to a non-local symmetry which is a linear combination of $\psi$ and the non-local symmetry generated from $\varphi$ by the recursion operator. Such solutions will still be non-invariant in the usual sense.

We shall choose $\varphi$ and $\psi$ as characteristics of point symmetries of $H C M A$. A general form of generators of point symmetries of $H C M A$ is obviously the same as for the elliptic $C M A$ obtained in [10]

$X=i\left(\Omega_{1} \partial_{2}-\Omega_{2} \partial_{1}-\Omega_{\overline{1}} \partial_{\overline{2}}+\Omega_{\overline{2}} \partial_{\overline{1}}\right)+C_{1}\left(z^{1} \partial_{1}+\bar{z}^{1} \partial_{\overline{1}}+u \partial_{u}\right)+i C_{2}\left(z^{2} \partial_{2}-\bar{z}^{2} \partial_{\overline{2}}\right)+H \partial_{u}$

where $C_{1}$ and $C_{2}$ are real constants and $\Omega\left(z^{i}, \bar{z}^{k}\right)$ and $H\left(z^{i}, \bar{z}^{k}\right)$ are arbitrary solutions of the linear system

$$
\Omega_{1 \overline{1}}=0, \quad \Omega_{2 \overline{2}}=0, \quad \Omega_{1 \overline{2}}=0, \quad \Omega_{2 \overline{1}}=0
$$

so that $\Omega=\omega\left(z^{i}\right)+\bar{\omega}\left(\bar{z}^{i}\right)$ and $H=h\left(z^{i}\right)+\bar{h}\left(\bar{z}^{i}\right)$. The corresponding symmetry characteristic [11] has the form

$$
\begin{aligned}
\widehat{\eta}= & i\left(u_{1} \Omega_{2}-u_{2} \Omega_{1}+u_{\overline{2}} \Omega_{\overline{1}}-u_{\overline{1}} \Omega_{\overline{2}}\right) \\
& +C_{1}\left(u-z^{1} u_{1}-\bar{z}^{1} u_{\overline{1}}\right)-i C_{2}\left(z^{2} u_{2}-\bar{z}^{2} u_{\overline{2}}\right)+H .
\end{aligned}
$$

Symmetries $\varphi$ and $\psi$ can be chosen as special cases of the expression (3.8).

In this paper we shall restrict ourselves only to the case $\psi=\varphi$ with the following two particular choices of partner symmetries: combined translational symmetries in $z^{1}, \bar{z}^{1}$ and $u$

$$
\psi=\varphi=u_{1}+u_{\overline{1}}+h\left(z^{2}\right)+\bar{h}\left(\bar{z}^{2}\right)
$$

and dilatational symmetries in the same variables

$$
\psi=\varphi=u-z^{1} u_{1}-\bar{z}^{1} u_{\overline{1}} .
$$




\section{Legendre transform of partner symmetries and ultra-hyperbolic metrics}

Let us perform the Legendre transformation of equations (2.7) and (3.4) to new variables

$$
v=u-z^{1} u_{1}-\bar{z}^{1} u_{\overline{1}}, \quad p=u_{1}, \quad \bar{p}=u_{\overline{1}}, \quad z^{1}=-v_{p}, \quad \bar{z}^{1}=-v_{\bar{p}}
$$

where $v=v\left(p, \bar{p}, z^{2}, \bar{z}^{2}\right)$. Then equation (2.7) in the new variables becomes

$$
v_{p \bar{p}} v_{2 \overline{2}}-v_{p \overline{2}} v_{\bar{p} 2}=v_{p \bar{p}}^{2}-v_{p p} v_{\bar{p} \bar{p}}
$$

and equations (3.4) together with their complex conjugates take the form

$$
\begin{aligned}
& \varphi_{p} v_{\bar{p} \bar{p}}-i \varphi_{\overline{2}} v_{p \bar{p}}-\varphi_{\bar{p}}\left(v_{p \bar{p}}-i v_{p \overline{2}}\right)=0 \\
& \varphi_{\bar{p}} v_{p p}+i \varphi_{2} v_{p \bar{p}}-\varphi_{p}\left(v_{p \bar{p}}+i v_{\bar{p} 2}\right)=0 \\
& \varphi_{p} \varphi_{\bar{p}}\left(2 v_{p \bar{p}}-v_{2 \overline{2}}\right)-\left(\varphi_{\bar{p}}^{2}+i \varphi_{\bar{p}} \varphi_{\overline{2}}\right) v_{p p}-\left(\varphi_{p}^{2}-i \varphi_{p} \varphi_{2}\right) v_{\bar{p} \bar{p}} \\
& +\left(\varphi_{2} \varphi_{\overline{2}}+i \varphi_{\overline{2}} \varphi_{p}-i \varphi_{2} \varphi_{\bar{p}}\right) v_{p \bar{p}}=0
\end{aligned}
$$

the last equation coinciding with its complex conjugate. Here we keep the same notation $\varphi$ for the Legendre transform of the symmetry characteristic which now depends on $p, \bar{p}$ instead of $z^{1}, \bar{z}^{1}$.

Ultra-hyperbolic metric (2.4) governed by the field equation (2.7) after Legendre transformation (4.1) becomes

$$
\begin{aligned}
& d s^{2}=\frac{1}{\left(v_{p p} v_{\bar{p} \bar{p}}-v_{p \bar{p}}^{2}\right)}\left[v_{p p}\left(v_{p \bar{p}} d p+v_{\bar{p} 2} d z^{2}\right)^{2}+v_{\bar{p} \bar{p}}\left(v_{p \bar{p}} d \bar{p}+v_{p \overline{2}} d \bar{z}^{2}\right)^{2}\right. \\
& \left.+\frac{\left(v_{p p} v_{\bar{p} \bar{p}}+v_{p \bar{p}}^{2}\right)}{v_{p \bar{p}}}\left|v_{p \bar{p}} d p+v_{\bar{p} 2} d z^{2}\right|^{2}\right]-\frac{\left(v_{p p} v_{\bar{p} \bar{p}}-v_{p \bar{p}}^{2}\right)}{v_{p \bar{p}}} d z^{2} d \bar{z}^{2}
\end{aligned}
$$

where we have used the Legendre transform (4.2) of $H C M A$ in the last term.

The Legendre transforms of translational and dilatational symmetries (3.9) and (3.10) become respectively

$$
\psi=\varphi=p+\bar{p}+h\left(z^{2}\right)+\bar{h}\left(\bar{z}^{2}\right)
$$

and

$$
\psi=\varphi=v
$$


With the choice (4.5) equations (4.3) become linear

$$
\begin{aligned}
& v_{\bar{p} \bar{p}}-\left(i \bar{h}^{\prime}\left(\bar{z}^{2}\right)+1\right) v_{p \bar{p}}+i v_{p \overline{2}}=0 \\
& v_{p p}+\left(i h^{\prime}\left(z^{2}\right)-1\right) v_{p \bar{p}}-i v_{\bar{p} 2}=0 \\
& 2 v_{p \bar{p}}-v_{2 \overline{2}}-\left(i \bar{h}^{\prime}\left(\bar{z}^{2}\right)+1\right) v_{p p}+\left(i h^{\prime}\left(z^{2}\right)-1\right) v_{\bar{p} \bar{p}} \\
& +\left[h^{\prime}\left(z^{2}\right) \bar{h}^{\prime}\left(\bar{z}^{2}\right)-i\left(h^{\prime}\left(z^{2}\right)-\bar{h}^{\prime}\left(\bar{z}^{2}\right)\right)\right] v_{p \bar{p}}=0 .
\end{aligned}
$$

This system has no nontrivial differential compatibility conditions since the equations $\left(v_{p \overline{2}}\right)_{2}=\left(v_{2 \overline{2}}\right)_{p},\left(v_{\bar{p} 2}\right)_{\overline{2}}=\left(v_{2 \overline{2}}\right)_{\bar{p}}$ and $\left(v_{p \overline{2}}\right)_{\bar{p} 2}=\left(v_{\bar{p} 2}\right)_{\overline{2} p}$ are satisfied identically.

With the choice (4.6) equations (4.3) are still non-linear

$$
\begin{aligned}
& v_{p} v_{\bar{p} \bar{p}}-i v_{\overline{2}} v_{p \bar{p}}-v_{\bar{p}}\left(v_{p \bar{p}}-i v_{p \overline{2}}\right)=0 \\
& v_{\bar{p}} v_{p p}+i v_{2} v_{p \bar{p}}-v_{p}\left(v_{p \bar{p}}+i v_{\bar{p} 2}\right)=0 \\
& \left(v_{2} v_{\overline{2}}+i v_{\overline{2}} v_{p}-i v_{2} v_{\bar{p}}+2 v_{p} v_{\bar{p}}\right) v_{p \bar{p}} \\
& -\left(v_{\bar{p}}^{2}+i v_{\bar{p}} v_{\overline{2}}\right) v_{p p}-\left(v_{p}^{2}-i v_{p} v_{2}\right) v_{\bar{p} \bar{p}}-v_{p} v_{\bar{p}} v_{2 \overline{2}}=0
\end{aligned}
$$

again with no nontrivial differential compatibility conditions.

Theorem 1 Solutions of the over-determined system (4.8) are functionally invariant, i.e. if $v$ is a solution to (4.8), then $f(v)$ is also a solution to (4.8) whenever $f$ is an arbitrary function $\in C^{2}$.

The proof follows from a substitution of $f(v)$ instead of $v$ in equations (4.8).

There is a particular class of solutions of the system (4.8) satisfying a linear over-determined system of six equations

$$
\begin{aligned}
v_{p \bar{p}} & =a v_{p}+\bar{a} v_{\bar{p}} \\
v_{p p} & =(\bar{a}+i \bar{b}) v_{p}-i \bar{a} v_{2} \\
v_{\bar{p} \bar{p}} & =(a-i b) v_{\bar{p}}+i a v_{\overline{2}} \\
v_{p \overline{2}} & =b v_{p}-i \bar{a} v_{\bar{p}}+\bar{a} v_{\overline{2}} \\
v_{\bar{p} 2} & =\bar{b} v_{\bar{p}}+i a v_{p}+a v_{2} \\
v_{2 \overline{2}} & =(a+i b) v_{p}+b v_{2}+(\bar{a}-i \bar{b}) v_{\bar{p}}+\bar{b} v_{\overline{2}}
\end{aligned}
$$

where $a$ and $b$ are arbitrary complex constants. Here the first three of these equations generate the last three equations as their differential compatibility conditions. 
The system (4.9) also has no nontrivial differential compatibility conditions, i.e. equations $\left(v_{p p}\right)_{\bar{p}}=\left(v_{p \bar{p}}\right)_{p},\left(v_{p \overline{2}}\right)_{2}=\left(v_{2} \overline{2}\right)_{p}$ and their complex conjugates are identically satisfied. The substitution of the second derivatives from (4.9) to (4.8) identically satisfies the latter equations.

General solution of the system (4.9) is obtained in the form

$$
v=\sum_{j=1}^{n} C_{j} \exp \left(\alpha_{j} p+\bar{\alpha}_{j} \bar{p}+\beta_{j} z^{2}+\bar{\beta}_{j} \bar{z}^{2}\right)
$$

where the sum over integer $j$ may contain finite or infinite number of terms, $C_{j}$ are arbitrary real constants and $\alpha_{j}$ and $\beta_{j}$ are determined by the equations

$$
\begin{gathered}
\left|\alpha_{j}\right|^{2}=a \alpha_{j}+\bar{a} \bar{\alpha}_{j}, \\
\beta_{j}=i \frac{\alpha_{j}^{2}-(\bar{a}+i \bar{b}) \alpha_{j}}{\bar{a}}
\end{gathered}
$$

together with the complex conjugate to (4.12). The first equation (4.11) is easily solved in polar coordinates $a=r e^{i \theta}, \bar{a}=r e^{-i \theta}, \alpha=\chi e^{i \mu}, \bar{\alpha}=\chi e^{-i \mu}$ in the form

$$
\chi=2 r \cos (\theta+\mu)
$$

where $\mu$ remains as a free parameter.

Substituting for $v$ in (4.4) any solution of the form (4.10) satisfying (4.13) and (4.12) we obtain explicitly an ultra-hyperbolic 'heavenly' [1 metric. Since generic solution of the form (4.10) obviously depends on four independent variables and hence is non-invariant, the resulting metric has no Killing vectors. The justification for this statement is given in section 7 where we examine the Killing equations for (4.4).

Though we have not found the general solution of the non-linear system (4.8), we essentially enlarge the class of its solutions using the property of functional invariance from theorem 1. Due to this property any smooth function of the solution (4.10) is again a solution of (4.8) though not of the system (4.9) since the latter system does not possess the above mentioned property. These more general solutions also can be used in the formula (4.4) giving explicitly more classes of 4-dimensional ultra-hyperbolic heavenly metrics admitting no Killing vectors.

To simplify the solution of the linear system (4.7), we will restrict ourselves to the linear function $h\left(z^{2}\right): h=\nu z^{2}, \bar{h}=\bar{\nu} \bar{z}^{2}$ where $\nu$ is an arbitrary 
constant, so that we obtain the linear system with constant coefficients

$$
\begin{aligned}
& v_{\bar{p} \bar{p}}-(i \bar{\nu}+1) v_{p \bar{p}}+i v_{p \overline{2}}=0 \\
& v_{p p}+(i \nu-1) v_{p \bar{p}}-i v_{\bar{p} 2}=0 \\
& {\left[2+|\nu|^{2}-i(\nu-\bar{\nu})\right] v_{p \bar{p}}-(i \bar{\nu}+1) v_{p p}+(i \nu-1) v_{\bar{p} \bar{p}}-v_{2 \overline{2}}=0 .}
\end{aligned}
$$

We note that the particular solution (4.10) of the system (4.8) satisfying (4.9) turns out to be also a particular solution of the system (4.14) with the additional constraint on the parameters in (4.9)

$$
\bar{a}=-a, \quad b=(\bar{\nu}-i) a
$$

where the second equation is just the relation between parameters of the two systems. The constraints (4.13) and (4.12) on the parameters in the solution (4.10) then take the form $(\theta=\pi / 2)$

$$
\chi=-2 r \sin \mu, \quad \beta=-i \frac{\alpha^{2}}{a}+\nu
$$

It is easy to find general solution of the linear system (4.14) with constant coefficients in the form similar to (4.10)

$$
v=\sum_{j=1}^{n} C_{j} \exp \left(\alpha_{j} p+\bar{\alpha}_{j} \bar{p}+\beta_{j} z^{2}+\bar{\beta}_{j} \bar{z}^{2}\right)
$$

where the sum over integer $j$ may again contain finite or infinite number of terms, $C_{j}$ are arbitrary real constants and $\beta_{j}$ are expressed through $\alpha_{j}$ by the relation

$$
\beta_{j}=\left(\nu+i-i \frac{\alpha_{j}}{\bar{\alpha}_{j}}\right) \alpha_{j}
$$

and its complex conjugate.

The substitution of any solution of the form (4.16) with (4.17) for $v$ in the formula (4.4) gives us explicitly another class of ultra-hyperbolic heavenly metrics. The solution (4.16) depends on four variables in the generic case, when the number of terms in the sum (4.16) is not less than four, and hence the corresponding metric has no Killing vectors. We shall prove this in section 7 by an examination of the Killing equations. 


\section{Symmetries and recursions of the second heavenly equation of Plebañski}

Let $\theta=\theta(w, z, x, y)$ be holomorphic complex-valued function of four complex variables in some local coordinate system on a complex 4-dimensional manifold $\mathcal{M}$. The heavenly metric of Plebañski [1] locally defined on $\mathcal{M}$ is defined by (we skip here an overall factor 2)

$$
d s^{2}=d w d x+d z d y-\theta_{x x} d z^{2}-\theta_{y y} d w^{2}+2 \theta_{x y} d w d z
$$

where subscripts denote partial derivatives with respect to corresponding variables. This metric is governed by the second heavenly equation [1]

$$
\theta_{x w}+\theta_{y z}+\theta_{x x} \theta_{y y}-\theta_{x y}^{2}=0
$$

for the potential $\theta$ in the metric (5.1).

\subsection{Recursion relations from the divergence form of the determining equation for symmetries}

The determining equation for symmetries of the heavenly equation (15.2) has the form

$$
\varphi_{x w}+\varphi_{y z}+\theta_{y y} \varphi_{x x}+\theta_{x x} \varphi_{y y}-2 \theta_{x y} \varphi_{x y}=0
$$

which is a linearization of (5.2). It can also be written as $\square_{\theta} \varphi=0$ with the operator $\square_{\theta}$ defined by

$$
\square_{\theta}=D_{x} D_{w}+D_{y} D_{z}+\theta_{y y} D_{x}^{2}+\theta_{x x} D_{y}^{2}-2 \theta_{x y} D_{x} D_{y}
$$

where $D_{x}, D_{w}, D_{y}, D_{z}$ denote total derivatives with respect to corresponding variables. We note that the determining equation (5.3) can be presented in the form of total divergence, i.e. differential conservation law

$$
D_{x}\left(\varphi_{w}+\theta_{y y} \varphi_{x}-\theta_{x y} \varphi_{y}\right)+D_{y}\left(\varphi_{z}+\theta_{x x} \varphi_{y}-\theta_{x y} \varphi_{x}\right)=0
$$

so that there locally exists a potential $\psi$ such that

$$
\psi_{y}=\varphi_{w}+\theta_{y y} \varphi_{x}-\theta_{x y} \varphi_{y}, \quad \psi_{x}=-\left(\varphi_{z}-\theta_{x y} \varphi_{x}+\theta_{x x} \varphi_{y}\right)
$$

and differential compatibility conditions $\left(\psi_{y}\right)_{x}=\left(\psi_{x}\right)_{y}$ for the system (5.6) coincide with the determining equation for symmetries (15.3). 
We introduce linear differential operators

$$
L_{y}=D_{w}+\theta_{y y} D_{x}-\theta_{x y} D_{y}, \quad L_{x}=-\left(D_{z}-\theta_{x y} D_{x}+\theta_{x x} D_{y}\right)
$$

so that the operator (5.4) takes the form

$$
\square_{\theta}=D_{x} L_{y}-D_{y} L_{x}=L_{y} D_{x}-L_{x} D_{y}
$$

and the relations (5.6) become

$$
\psi_{y}=L_{y} \varphi, \quad \psi_{x}=L_{x} \varphi
$$

The commutator of the two operators has the form

$$
\left[L_{x}, L_{y}\right]=\left(\theta_{x w}+\theta_{y z}+\theta_{x x} \theta_{y y}-\theta_{x y}^{2}\right)_{y} D_{x}-\left(\theta_{x w}+\theta_{y z}+\theta_{x x} \theta_{y y}-\theta_{x y}^{2}\right)_{x} D_{y}
$$

and hence $\left[L_{x}, L_{y}\right]=0$ on the solution manifold of the second heavenly equation (5.2). Alternatively, vanishing of the commutator of $L_{x}$ and $L_{y}$ reproduces the equation

$$
\theta_{x w}+\theta_{y z}+\theta_{x x} \theta_{y y}-\theta_{x y}^{2}=C_{w}(w, z)
$$

where $C(w, z)$ an arbitrary function of $w$ and $z$ which after redefining $\theta$ to $\theta+x C(w, z)$ coincides with the original equation (5.2). Note that this redefinition does not change the equations (5.3) - (5.9) .

Let $\varphi$ be a symmetry of (5.2) so that it satisfies the determining equation (5.3) $\square_{\theta} \varphi=0$ and $\psi$ be a corresponding potential for $\varphi$ related to it by formulas (5.9). Then a simple calculation shows that $\psi$ is also a symmetry, i.e. it satisfies the same equation (5.3)

$$
\square_{\theta} \psi=L_{y} \psi_{x}-L_{x} \psi_{y}=-\left[L_{x}, L_{y}\right] \varphi=0
$$

where we have substituted $\psi_{x}$ and $\psi_{y}$ from (5.9) and used the commutativity of $L_{x}$ and $L_{y}$ on the solution manifold of (5.2). Therefore, the potential $\psi$ for any symmetry $\varphi$ is also a symmetry and hence the equations (5.9) are recursion relations for partner symmetries of (5.2).

The Lax pair of Mason and Newman for the equation (5.2) [12, 13] can be expressed through $L_{x}$ and $L_{y}$ as

$$
L_{0}=D_{y}-\lambda L_{y}, \quad L_{1}=D_{x}-\lambda L_{x}
$$


so that $\left[L_{0}, L_{1}\right]=\lambda^{2}\left[L_{y}, L_{x}\right]$ and the vanishing of the commutator $\left[L_{0}, L_{1}\right]$ reproduces the equation (5.2) up to redefinition of $C(w, z)$, same as for $\left[L_{x}, L_{y}\right]=0$.

Recursion relations (5.9) for symmetries can be expressed in terms of non-local recursion operator $R$ defined by

$$
\psi=R \varphi=D_{y}^{-1} L_{y} \varphi, \quad \psi=R \varphi=D_{x}^{-1} L_{x} \varphi .
$$

\subsection{Point symmetries of the second heavenly equation}

Here again we will avoid operating explicitly with non-local symmetries and use instead the equations (5.6) only with the point partner symmetries $\varphi$ and $\psi$. Then these equations are equivalent to an invariance condition for solutions of the second heavenly equation with respect to non-local symmetry which is a linear combination of $\psi$ and the non-local symmetry generated from $\varphi$ by the recursion operator. Such solutions will still be non-invariant in the usual sense.

Though here we use only an obvious translational symmetry for this purpose, it is convenient for future analysis in the same framework to present explicitly basis generators of the complete symmetry algebra of point symmetries for the second heavenly equation (5.2). Symmetry generators of its one-parameter subgroups have the form

$$
\begin{aligned}
& X_{1}=\partial / \partial x, \quad X_{2}=2 z \partial / \partial x-x y \partial / \partial \theta, \quad X_{3}=y \partial / \partial y+w \partial / \partial w+\theta \partial / \partial \theta \\
& X_{4}=x \partial / \partial x+y \partial / \partial y+3 \theta \partial / \partial \theta, \quad Y_{a}=\left(y a_{w}-x a_{z}\right) \partial / \partial \theta, \quad H_{d}=\partial / \partial \theta \\
& Z_{b}=b_{w} \partial / \partial x+b_{z} \partial / \partial y+(1 / 2)\left(x^{2} b_{z z}+y^{2} b_{w w}-2 x y b_{z w}\right) \partial / \partial \theta \quad(5.12) \\
& G_{c}=\left(x c_{z w}-y c_{w w}\right) \partial / \partial x+\left(x c_{z z}-y c_{z w}\right) \partial / \partial y+c_{w} \partial / \partial z-c_{z} \partial / \partial w \\
& +(1 / 6)\left(x^{3} c_{z z z}-3 x^{2} y c_{z z w}+3 x y^{2} c_{z w w}-y^{3} c_{w w w}\right) \partial / \partial \theta
\end{aligned}
$$

where $a(z, w), b(z, w), c(z, w)$ and $d(z, w)$ are arbitrary functions of $z, w$. Since some of the generators contain arbitrary functions, the total symmetry group is an infinite Lie (pseudo)group.

Of course, some of these symmetries are quite obvious, such as translations in all independent variables and combined dilatations

$$
y^{\prime}=\lambda y, \quad w^{\prime}=\lambda w, \quad \theta^{\prime}=\lambda \theta \quad \text { and } \quad x^{\prime}=\lambda x, \quad y^{\prime}=\lambda y, \quad \theta^{\prime}=\lambda^{3} \theta
$$

generated by $X_{3}$ and $X_{4}$ respectively. From symmetry considerations one may wonder where is the generator of the uniform dilatations in $x, z, \theta: \tilde{X}_{3}=$ 
$x \partial / \partial x+z \partial / \partial z+\theta \partial / \partial \theta$ ? The answer is that for a particular choice $c=z w$ the generator $G_{z w}$ reduces to another generator of dilatations

$$
G_{z w}=x \partial / \partial x-y \partial / \partial y+z \partial / \partial z-w \partial / \partial w
$$

so that $\tilde{X}_{3}=G_{z w}+X_{3}$. Seemingly missing generators of translations in $y, z, w$ can also be found in (5.12) for certain special choices of $b$ and $c$ as

$$
Z_{z}=\partial / \partial y, \quad G_{w}=\partial / \partial z, \quad G_{-z}=\partial / \partial w .
$$

We also have the generator of simultaneous rotations in $(x, y)$ and $(w, z)$ complex planes

$$
G_{c}=x \partial / \partial y-y \partial / \partial x+w \partial / \partial z-z \partial / \partial w
$$

which is obtained from $G_{c}$ by the choice of $c=\left(z^{2}+w^{2}\right) / 2$.

In the next section we shall use only one of the simplest translational symmetries from (5.12), namely $G_{z}=-\partial / \partial w$ with the characteristic $\theta_{w}$, and still obtain very non-trivial results.

For completeness we present a table of commutators of the generators (5.12) where the commutator $\left[X_{i}, X_{j}\right]$ stands at the intersection of $i$ th row and $j$ th column. It is convenient to introduce for this table the following shorthand notation for a skew-symmetric differentiation of a pair of functions $a(z, w)$ and $b(z, w): a \wedge b=a_{z} b_{w}-b_{z} a_{w}$ and $s=x w+y z$, so that $x a_{z}-y a_{w}=$ $a \wedge s$ and $x(f \wedge b)_{z}-y(f \wedge b)_{w}=(f \wedge b) \wedge s$. We also denote $\hat{c}_{z}=z c_{z}-c$ and $\hat{c}_{w}=w c_{w}-c$. In table $1 a, b, c, d, e, f, g, h$ are arbitrary functions of $z, w$.

\begin{tabular}{|c|c|c|c|c|c|c|c|c|}
\hline & $X_{1}$ & $X_{2}$ & $X_{3}$ & $X_{4}$ & $Y_{a}$ & $Z_{b}$ & $G_{c}$ & $H_{d}$ \\
\hline$X_{1}$ & 0 & $-Y_{w}$ & 0 & $X_{1}$ & $-H_{a_{z}}$ & $-Y_{b_{z}}$ & $Z_{c_{z}}$ & 0 \\
\hline$X_{2}$ & $Y_{w}$ & 0 & 0 & $X_{2}$ & $-2 H_{z a_{z}}$ & $-Y_{2 \hat{b}_{z}+b}$ & $2 Z_{\hat{c}_{z}}$ & 0 \\
\hline$X_{3}$ & 0 & 0 & 0 & 0 & $-H_{\left(w a_{w}\right) \wedge s}$ & $Z_{\hat{b}_{w}}$ & $G_{\hat{c}_{w}}$ & $H_{\hat{d}_{w}}$ \\
\hline$X_{4}$ & $-X_{1}$ & $-X_{2}$ & 0 & 0 & $2 H_{a \wedge s}$ & $-Z_{b}$ & 0 & $-3 H_{d}$ \\
\hline$Y_{e}$ & $H_{e_{z}}$ & $2 H_{z e_{z}}$ & $H_{\left(w e_{w}\right) \wedge s}$ & $-2 H_{e \wedge s}$ & 0 & $H_{e \wedge b}$ & $H_{(e \wedge c) \wedge s}$ & 0 \\
\hline$Z_{f}$ & $Y_{f_{z}}$ & $Y_{2 \hat{f}_{z}+f}$ & $-Z_{\hat{f}_{w}}$ & $Z_{f}$ & $-H_{a \wedge f}$ & $-H_{(f \wedge b) \wedge s}$ & $Z_{c \wedge f}$ & 0 \\
\hline$G_{g}$ & $-Z_{g_{z}}$ & $-2 Z_{\hat{g}_{z}}$ & $-G_{\hat{g}_{w}}$ & 0 & $-H_{(a \wedge g) \wedge s}$ & $-Z_{g \wedge b}$ & $G_{c \wedge g}$ & $H_{d \wedge g}$ \\
\hline$H_{h}$ & 0 & 0 & $-H_{\hat{h}_{w}}$ & $3 H_{h}$ & 0 & 0 & $-H_{h \wedge c}$ & 0 \\
\hline
\end{tabular}

Table 1: Commutators of point symmetries of the second heavenly equation.

All contact symmetries of the second heavenly equation (5.2) coincide with its prolonged point symmetries.

Symmetries of the second heavenly equation were also studied in [9, 14. 


\subsection{Legendre transformation and heavenly metric}

Our final goal is to end up with linear equations. This will be achieved in the next section by applying the partial Legendre transformation

$$
u=\theta-w \theta_{w}-y \theta_{y}, \quad \theta_{w}=t, \quad \theta_{y}=r, \quad w=-u_{t}, \quad y=-u_{r}
$$

to second heavenly equation (5.2) together with equations (5.6) for some choices of local symmetries $\varphi$ and $\psi$. The existence condition for Legendre transformation (5.13) has the form

$$
\Delta=u_{t t} u_{r r}-u_{r t}^{2} \neq 0 .
$$

The Legendre transform of (15.2) is

$$
u_{t t}\left(u_{x x}+u_{r z}\right)+u_{x t}\left(u_{r r}-u_{x t}\right)-u_{r t}\left(u_{r x}+u_{t z}\right)=0 .
$$

The Legendre transformation (5.13) of the metric (5.1) results in

$$
\begin{aligned}
& d s^{2}=\frac{\left[u_{t t}\left(u_{t t} d t+u_{t r} d r+u_{t x} d x+u_{t z} d z\right)+\left(u_{t t} u_{r x}-u_{t r} u_{t x}\right) d z\right]^{2}}{u_{t t}\left(u_{t t} u_{r r}-u_{t r}^{2}\right)} \\
& -\frac{\left(u_{t t} u_{x x}-u_{t x}^{2}\right)}{u_{t t}} d z^{2}-\left(u_{t t} d t+u_{t r} d r+u_{t x} d x+u_{t z} d z\right) d x \\
& -\left(u_{r t} d t+u_{r r} d r+u_{r x} d x+u_{r z} d z\right) d z
\end{aligned}
$$

with the potential $u$ satisfying the equation (15.15).

\section{Example: use of translational symmetries}

\subsection{Case of equal symmetries}

At first we consider the case when the two partner symmetries are equal to each other $\varphi=\psi$ and we choose $\varphi$ to be equal to the characteristic of the translational symmetry $\varphi=\theta_{w}$. Then the equations (5.6) take the form

$$
\begin{array}{r}
-\theta_{y w}+\theta_{w w}+\theta_{y y} \theta_{w x}-\theta_{x y} \theta_{w y}=0 \\
\theta_{x w}+\theta_{w z}+\theta_{x x} \theta_{w y}-\theta_{x y} \theta_{w x}=0
\end{array}
$$

so that together with (5.2) we obtain a system of three equations. 
After applying Legendre transformation (5.13) equation (6.1) is linearized in the form

$$
u_{r t}+u_{r r}-u_{x t}=0
$$

and with the aid of the equation (6.3) the equations (6.2), (5.2) become respectively

$$
\begin{aligned}
& u_{r t}\left(u_{x x}+u_{r z}\right)-u_{r r}\left(u_{r x}+u_{x t}+u_{t z}\right)=0 \\
& -u_{t t}\left(u_{x x}+u_{r z}\right)+u_{r t}\left(u_{r x}+u_{x t}+u_{t z}\right)=0 .
\end{aligned}
$$

Solving the system (6.4), (6.5) algebraically we obtain two linear equations

$$
\begin{gathered}
u_{x x}+u_{r z}=0, \\
u_{r x}+u_{x t}+u_{t z}=0
\end{gathered}
$$

since the determinant of this system is non-zero due to the condition $\Delta \neq 0$ with $\Delta$ defined in (5.14).

The system of the three linear equations (6.3), (6.6) and (6.7) corresponds to the original second heavenly equation (5.2) plus two differential constraints (6.1) and (6.2).

Solution of this linear system has the form

$$
u=\sum_{j=1}^{n} C_{j} \exp \left(\alpha_{j} t+\beta_{j} r+\gamma_{j} x+\delta_{j} z\right)
$$

where $C_{j}$ are arbitrary constants and the parameters satisfy the relations

$$
\alpha_{j}=\frac{\beta_{j}^{2}}{\gamma_{j}-\beta_{j}}, \quad \delta_{j}=-\frac{\gamma_{j}^{2}}{\beta_{j}} .
$$

Substitution of this solution to the Legendre transform (5.16) of the heavenly metric (5.1) gives an explicit form of such a metric. The solution (6.8) depends on four variables in the generic case, when the number of terms in the sum (6.8) is greater than three, and hence the corresponding metric has no Killing vectors.

\subsection{Solutions invariant with respect to higher symmetry}

Here we consider the case when $\varphi$ is the translational symmetry $\varphi=\theta_{w}$ and its partner symmetry characteristic $\psi$ is equal to zero $\psi=0$. This means the 
invariance of the solution for $\theta$ with respect to the nonlocal higher symmetry $\psi$ generated from $\varphi=\theta_{w}$ by the recursion relations (5.6)

$$
\begin{gathered}
L_{y} \varphi=\theta_{w w}+\theta_{y y} \theta_{w x}-\theta_{x y} \theta_{w y}=0 \\
-L_{x} \varphi=\theta_{w z}-\theta_{x y} \theta_{w x}+\theta_{x x} \theta_{w y}=0
\end{gathered}
$$

so that we have again the system of three equations (6.10), (6.11) and (5.2).

Next we perform the partial Legendre transformation (5.13) of this system. The Legendre transform of (6.10) is

$$
u_{r r}-u_{t x}=0
$$

With the use of (6.12) the Legendre transforms of (6.11) and (5.2) become respectively

$$
\begin{aligned}
& u_{r r}\left(u_{r x}+u_{t z}\right)-u_{t r}\left(u_{r z}+u_{x x}\right)=0 \\
& u_{r t}\left(u_{r x}+u_{t z}\right)-u_{t t}\left(u_{r z}+u_{x x}\right)=0 .
\end{aligned}
$$

Due to the condition $\Delta \neq 0$ in (5.14) equations (6.13) and (6.14) become

$$
\begin{aligned}
& u_{r x}+u_{t z}=0 \\
& u_{r z}+u_{x x}=0
\end{aligned}
$$

so that we end up with the system of linear equations (6.12), (6.15) and (6.16).

Solution of this linear system has again the form (6.8) but with the modified relations between parameters

$$
\alpha_{j}=\frac{\beta_{j}^{2}}{\gamma_{j}}, \quad \delta_{j}=-\frac{\gamma_{j}^{2}}{\beta_{j}} .
$$

The corresponding explicit form of heavenly metric is obtained by a substitution of the solution (6.8) for $u$ into the formula (5.16). This metric generically has no Killing vectors for the same reason as mentioned above.

\section{Relationship between Killing vectors and symmetries of the potential}

Here we shall give proof of the non-existence of any Killing vectors for our metrics. 
Our emphasis will be on the existence problem. Thus we shall make use of the fact that a vector is an invariant object and the existence of a vector field in one frame implies its existence in any other frame. We have started out with the simple looking Kähler metric with the complicated $C M A$ equation as the condition for Ricci-flatness and after a Legendre transformation arrived at a complicated form of the metric with linear field equations which are easily solved. With the Killing vector there is a similar situation. Killing's equations are simple in the Kähler form of the metric and we obtain a linear first-order PDE, see (7.5) below, which encodes all the information in the Killing equations. On solutions of the field equations the Legendre transformation induces point transformations between the coordinates entering into the metric. This is a linear homogeneous transformation between the components of the Killing vector and its non-existence in one frame will imply its non-existence in any other frame.

\subsection{Analysis of the Killing equations for the Kähler metric}

Thus we consider first the Kähler metric (2.4) and let

$$
\vec{v}=\xi^{k}(z, \bar{z}) \frac{\partial}{\partial z^{k}}+\xi^{\bar{k}}(z, \bar{z}) \frac{\partial}{\partial \bar{z}^{k}}
$$

be the Killing vector for (2.4) where $z=\left(z^{1}, z^{2}\right)$ and $\bar{z}=\left(\bar{z}^{1}, \bar{z}^{2}\right)$ and summation over dummy indices ranges over two values for both barred and unbarred indices. The reality condition for $\vec{v}$ implies $\xi^{\bar{k}}=\xi^{k}$.

The Killing equations for the metric (2.4) fall into two sets

$$
u_{i \bar{k}} \xi_{j}^{\bar{k}}=0, \quad u_{k \bar{j}} \xi_{\bar{i}}^{k}=0
$$

and

$$
\left(\xi^{k} u_{k \bar{j}}\right)_{i}+\left(\xi^{\bar{k}} u_{\bar{k} i}\right)_{\bar{j}}=0
$$

where subscripts denote partial derivatives. The determinant of the linear equations (7.2) is non-zero due to the $C M A$ equation (2.3)

$$
\operatorname{det}\left(u_{i \bar{k}}\right)=u_{1 \overline{1}} u_{2 \overline{2}}-u_{2 \overline{1}} u_{1 \overline{2}}= \pm 1
$$

and hence these equations have only vanishing solutions $\xi_{j}^{\bar{k}}=0, \xi_{\bar{i}}^{k}=0$, so that

$$
\xi^{i}=\xi^{i}(z), \quad \xi^{\bar{i}}=\xi^{\bar{i}}(\bar{z}) .
$$


The remaining Killing equations become

$$
\left(\xi^{k} u_{k}+\xi^{\bar{k}} u_{\bar{k}}\right)_{i \bar{j}}=0
$$

with the solution

$$
u_{k} \xi^{k}(z)+u_{\bar{k}} \xi^{\bar{k}}(\bar{z})=h(z)+\bar{h}(\bar{z})
$$

where $h$ is an arbitrary biholomorphic function.

Hence the Killing equations are equivalent to the linear equation (17.5) for $\xi^{k}(z), \xi^{\bar{k}}(\bar{z})$ which should be satisfied for a given solution $u(z, \bar{z})$ of $C M A$ if a Killing vector for the corresponding metric (2.4) exists.

We note that if $\xi^{k}, \xi^{\bar{k}}$ are chosen as coefficients of a generator of point symmetries (3.7) with $C_{1}=0$, then (7.5) coincides with the invariance condition $\hat{\eta}=0$ for solutions of $C M A$, where $\hat{\eta}$ is the symmetry characteristic (3.8). More generally (7.5) determines conditionally invariant solutions of $C M A$ [15], if conditional symmetries exist. Thus, any such symmetry in a solution of $C M A$ implies the existence of a Killing vector for the Kähler metric (2.4).

The Legendre transformation (4.1) induces an invertible point coordinate transformation

$$
p=u_{1}(z, \bar{z}), \quad \bar{p}=u_{\overline{1}}(z, \bar{z}), \quad z^{1}=-v_{p}\left(p, \bar{p}, z^{2}, \bar{z}^{2}\right), \quad \bar{z}^{1}=-v_{\bar{p}}\left(p, \bar{p}, z^{2}, \bar{z}^{2}\right)
$$

on solutions $u(z, \bar{z})$ and $v\left(p, \bar{p}, z^{2}, \bar{z}^{2}\right)$ of $H C M A(2.7)$ and its Legendre transform (4.2) respectively and $z^{2}, \bar{z}^{2}$ are not transformed. Under this transformation the condition (7.5), equivalent to the Killing equations, results in

$$
\begin{aligned}
& p \xi^{1}\left(-v_{p}, z^{2}\right)+v_{2} \xi^{2}\left(-v_{p}, z^{2}\right)+\bar{p} \xi^{\overline{1}}\left(-v_{\bar{p}}, \bar{z}^{2}\right)+v_{\overline{2}} \xi^{\overline{2}}\left(-v_{\bar{p}}, \overline{z^{2}}\right) \\
& =h\left(-v_{p}, z^{2}\right)+\bar{h}\left(-v_{\bar{p}}, \bar{z}^{2}\right)
\end{aligned}
$$

We should account also for a transformation of components of the Killing vectors induced by the transformation (7.6). Let

$$
\vec{v}=\eta^{p} \frac{\partial}{\partial p}+\eta^{\bar{p}} \frac{\partial}{\partial \bar{p}}+\eta^{2} \frac{\partial}{\partial z^{2}}+\eta^{\overline{2}} \frac{\partial}{\partial \bar{z}^{2}}
$$

be the Killing vector (17.1) in the frame transformed by (17.6). The transformation law for the components of the Killing vector is given by

$$
\begin{array}{ll}
\xi^{1}=-\left(\eta^{p} v_{p p}+\eta^{\bar{p}} v_{p \bar{p}}+\eta^{2} v_{p 2}+\eta^{\overline{2}} v_{p \overline{2}}\right), & \xi^{2}=\eta^{2} \\
\xi^{\overline{1}}=-\left(\eta^{p} v_{\bar{p} p}+\eta^{\bar{p}} v_{\bar{p} \bar{p}}+\eta^{2} v_{\bar{p} 2}+\eta^{\overline{2}} v_{\bar{p} \overline{2}}\right), & \xi^{\overline{2}}=\eta^{\overline{2}}
\end{array}
$$


where the arguments of $\eta^{p}, \eta^{2}, \eta^{\bar{p}}, \eta^{\overline{2}}$ and second derivatives of $v$ on the right hand sides of (7.9) consist of $p, \bar{p}, z^{2}, \bar{z}^{2}$. Hence the existence of $\vec{v}$ defined by (17.1) is equivalent to that of $\vec{v}$ defined by (7.8). Given some solution $v$ of (4.2), a Killing vector for the transformed metric (4.4) exists only if one can satisfy (7.7).

We need to check if this equation can be satisfied by our solutions (4.10) and (4.16). Both of them have the form

$$
v=\sum_{j=1}^{n} C_{j} e^{\Phi_{j}}
$$

where $C_{j}$ are arbitrary real constants,

$$
\Phi_{j}=\alpha_{j} p+\bar{\alpha}_{j} \bar{p}+\beta_{j} z^{2}+\bar{\beta}_{j} \bar{z}^{2}
$$

and the parameters $\alpha_{j}, \beta_{j}$ satisfy the conditions (4.11), (4.12) for (4.10) and (4.17) for (4.16).

Let $n \geq 4$ in (7.10) and $\Phi_{1}, \Phi_{2}, \Phi_{3}, \Phi_{4}$ be linearly independent, i.e. the transformation (17.11) from $p, \bar{p}, z^{2}, \bar{z}^{2}$ to $\Phi_{1}, \Phi_{2}, \Phi_{3}, \Phi_{4}$ is invertible, provided that $\alpha_{1} \alpha_{2} \alpha_{3} \alpha_{4} \neq 0$ and the determinant of the matrix of coefficients of (7.11) is non-zero

$$
\left|\begin{array}{cccc}
1 & e^{-2 i \mu_{1}} & e^{2 i \mu_{1}} & e^{-4 i \mu_{1}} \\
1 & e^{-2 i \mu_{2}} & e^{2 i \mu_{2}} & e^{-4 i \mu_{2}} \\
1 & e^{-2 i \mu_{3}} & e^{2 i \mu_{3}} & e^{-4 i \mu_{3}} \\
1 & e^{-2 i \mu_{4}} & e^{2 i \mu_{4}} & e^{-4 i \mu_{4}}
\end{array}\right| \neq 0
$$

where $\mu_{j}$ are the phases of $\alpha_{j}$. The same condition holds for both solutions (4.10) and (4.16). Then $p, \bar{p}, z^{2}, \bar{z}^{2}$ can be expressed through $\Phi_{1}, \Phi_{2}, \Phi_{3}, \Phi_{4}$ and the same for $\Phi_{5}, \ldots, \Phi_{n}$, so that $\Phi_{j}$ for $j=1,2,3,4$ can be chosen as new independent variables in (7.7) and the equation (7.7) takes the form

$$
G\left(\Phi_{1}, \Phi_{2}, \Phi_{3}, \Phi_{4}\right)=0
$$

Obviously the equation (17.7) cannot be satisfied identically for any solution $v$ of (4.2) just by a suitable choice of of functions $\xi^{i}, \xi^{\bar{i}}, h, \bar{h}$ because of $p, \bar{p}, v_{2}, v_{\overline{2}}$ entering explicitly its coefficients. A similar remark applies also to the original equation (7.5) where generically the coefficients $u_{i}, u_{\bar{i}}$ depend on $z$ and $\bar{z}$ together while the unknowns $\xi^{i}, \xi^{\bar{i}}$ and $h, \bar{h}$ depend only on $z$ or $\bar{z}$ separately. Hence (17.5) and (7.7) should be considered as the equations determining particular solutions of $C M A$ or (4.2) respectively for any choice of 
$\xi^{i}, \xi^{\bar{i}}, h, \bar{h}$ and this choice is constrained by compatibility conditions of (17.5) with $C M A$ and (7.7) with (4.2).

In our case solutions of (4.2) are already determined in (7.10) up to arbitrary constants by solving second-order linear equations together with the Legendre-transformed $H C M A$ and hence, having no functional arbitrariness, they cannot satisfy in addition the first-order equation (7.7). Thus for any choice of $\xi^{i}, \xi^{\bar{i}}, h, \bar{h}$ (7.7) is not an identity but an equation of the form (7.13). This implies a dependence of the independent variables which is a contradiction that proves nonexistence of the Killing vectors for the metric (4.4) where the potential $v$ is determined by (4.10) and (4.16) with $n \geq 4$ and the condition (7.12) is satisfied.

If $n \leq 3$ then this reasoning obviously does not work and Killing vectors may exist.

Thus we have proved the following theorem.

Theorem 2 Metric (4.4) with $v$ defined either by (4.10) with the conditions (4.11), 4.12), or by (4.16) with the conditions (4.17) provided that $n \geq 4$, $\alpha_{1} \alpha_{2} \alpha_{3} \alpha_{4} \neq 0$ and satisfying the non-degeneracy condition (7.12) admits no Killing vectors.

\subsection{Analysis of the Killing equations for the second heavenly metric}

Now we shall perform a similar analysis for the heavenly metric (5.1) governed by the potential satisfying the second heavenly equation (5.2). Let

$$
\vec{\Omega}=\xi^{x}(x, y, z, w) \frac{\partial}{\partial x}+\xi^{y}(x, y, z, w) \frac{\partial}{\partial y}+\xi^{z}(x, y, z, w) \frac{\partial}{\partial z}+\xi^{w}(x, y, z, w) \frac{\partial}{\partial w}
$$

denote the Killing vector for the metric (5.1). The Killing equations for this metric fall into three sets

$$
\begin{aligned}
& \xi_{x}^{w}=0, \quad \xi_{y}^{z}=0, \quad \xi_{x}^{z}+\xi_{y}^{w}=0, \quad \xi_{x}^{y}+\xi_{z}^{w}-2 \theta_{x x} \xi_{x}^{z}=0 \\
& \xi_{x}^{x}+\xi_{w}^{w}+2 \theta_{x y} \xi_{x}^{z}=0, \quad \xi_{y}^{y}+\xi_{z}^{z}+2 \theta_{x y} \xi_{y}^{w}=0, \quad \xi_{y}^{x}+\xi_{w}^{z}-2 \theta_{y y} \xi_{y}^{w}=0 \\
& \xi^{x} \theta_{x x x}+\xi^{y} \theta_{y x x}+\xi^{z} \theta_{z x x}+\xi^{w} \theta_{w x x}=\xi_{z}^{y}-2 \theta_{x x} \xi_{z}^{z}+2 \theta_{x y} \xi_{z}^{w} \\
& \xi^{x} \theta_{x y y}+\xi^{y} \theta_{y y y}+\xi^{z} \theta_{z y y}+\xi^{w} \theta_{w y y}=\xi_{w}^{x}+2 \theta_{x y} \xi_{w}^{z}-2 \theta_{y y} \xi_{w}^{w} \\
& 2\left(\xi^{x} \theta_{x x y}+\xi^{y} \theta_{y x y}+\xi^{z} \theta_{z x y}+\xi^{w} \theta_{w x y}\right)+2 \theta_{x y}\left(\xi_{z}^{z}+\xi_{w}^{w}\right) \\
& -2 \theta_{x x} \xi_{w}^{z}-2 \theta_{y y} \xi_{z}^{w}+\xi_{w}^{y}+\xi_{z}^{x}=0
\end{aligned}
$$


of seven, two and one equations respectively. The first subsystem (7.15) is easily integrated to give

$$
\begin{aligned}
& \xi^{x}=-2 a \theta_{y}-x d_{w}-y b_{w}-e, \quad \xi^{y}=2 a \theta_{x}-x d_{z}-y b_{z}+c \\
& \xi^{z}=a x+b, \quad \xi^{w}=-a y+d
\end{aligned}
$$

where $a$ is an arbitrary constant and $b, c, d, e$ are arbitrary functions of $z, w$ only. The two equations (17.16) are integrated with respect to $x$ and $y$ respectively and the results are substituted into (17.17) which determines $y$ - and $x$-dependences of the integration "constants". Then we solve two remaining equations (7.16) algebraically with respect to $\left(\xi^{x} \theta_{x}+\xi^{y} \theta_{y}+\xi^{z} \theta_{z}+\xi^{w} \theta_{w}\right)_{x}$ and $\left(\xi^{x} \theta_{x}+\xi^{y} \theta_{y}+\xi^{z} \theta_{z}+\xi^{w} \theta_{w}\right)_{y}$ and then equate their cross-derivatives in $y$ and $x$ which gives additional constraints on the right-hand sides of these equations. In particular, we obtain that

$$
b=q_{w}+k z, \quad d=-q_{z}+k w
$$

where $k$ is an arbitrary constant and $q$ is an arbitrary function of $z, w$.

For the purpose of further integration we present the second heavenly equation (5.2) in a divergence form

$$
\left(\theta_{y} \theta_{x x}-\theta_{x} \theta_{y x}+2 \theta_{z}\right)_{y}=\left(\theta_{y} \theta_{x y}-\theta_{x} \theta_{y y}-2 \theta_{w}\right)_{x}
$$

so that we can locally define the potential $V$

$$
V_{x}=\theta_{y} \theta_{x x}-\theta_{x} \theta_{y x}+2 \theta_{z}, \quad V_{y}=\theta_{y} \theta_{x y}-\theta_{x} \theta_{y y}-2 \theta_{w}
$$

up to an arbitrary term depending only on $z, w$. Then we can further integrate the two equations (7.16), whose left-hand sides after the first integration were $\left(\xi^{x} \theta_{x}+\xi^{y} \theta_{y}+\xi^{z} \theta_{z}+\xi^{w} \theta_{w}\right)_{x}$ and $\left(\xi^{x} \theta_{x}+\xi^{y} \theta_{y}+\xi^{z} \theta_{z}+\xi^{w} \theta_{w}\right)_{y}$, with respect to $x$ and $y$ which results in

$$
\begin{aligned}
& \xi^{x} \theta_{x}+\xi^{y} \theta_{y}+\xi^{z} \theta_{z}+\xi^{w} \theta_{w} \equiv\left[x\left(q_{z w}-k\right)-y q_{w w}-e\right] \theta_{x} \\
& +\left[x q_{z z}-y\left(q_{z w}+k\right)+c\right] \theta_{y}+\left(a x+k z+q_{w}\right) \theta_{z}+\left(k w-a y-q_{z}\right) \theta_{w} \\
& =2(a V-k \theta)+\frac{1}{6}\left(x^{3} q_{z z z}-3 x^{2} y q_{z z w}+3 x y^{2} q_{z w w}-y^{3} q_{w w w}\right) \\
& +\frac{1}{2}\left[x^{2} c_{z}+x y\left(e_{z}-c_{w}\right)-y^{2} e_{w}\right]+x \rho+y \sigma+\kappa
\end{aligned}
$$

where we have used the expressions (7.18) for $\xi^{x}, \xi^{y}, \xi^{z}, \xi^{w}$ in the left-hand side of this equation and $\rho, \sigma, \kappa$ are new arbitrary functions of $z, w$. Hence the 
Killing equations (7.15) - (7.17) are equivalent to the linear first-order PDE (7.22) which should be identically satisfied for a given solution of the heavenly equation (5.2) with a suitable choice of arbitrary functions $q, c, e, \rho, \sigma, \kappa$ of the variables $z, w$, arbitrary constants $a, k$ and the integration "constant" for the potential $V$ in (7.21) depending on $z, w$. If (7.22) is satisfied then the components of the Killing vector are given by (17.18) together with (7.19).

The Legendre transformation (5.13) induces an invertible point coordinate transformation

$$
r=\theta_{y}(x, y, z, w), t=\theta_{w}(x, y, z, w), \quad y=-u_{r}(x, r, z, t), w=-u_{t}(x, r, z, t)
$$

on solutions $\theta(x, y, z, w)$ and $u(x, r, z, t)$ of the second heavenly equation (5.2) and its Legendre transform (5.15) respectively and $x, z$ are not transformed. Let

$$
\vec{\Omega}=\eta^{x} \frac{\partial}{\partial x}+\eta^{r} \frac{\partial}{\partial r}+\eta^{z} \frac{\partial}{\partial z}+\eta^{t} \frac{\partial}{\partial t}
$$

be a Killing vector (7.14) in the frame transformed by (7.23). The components of the Killing vector are accordingly transformed by

$$
\begin{array}{ll}
\xi^{y}=-\left(\eta^{x} u_{r x}+\eta^{r} u_{r r}+\eta^{z} u_{r z}+\eta^{t} u_{r t}\right), & \xi^{x}=\eta^{x} \\
\xi^{w}=-\left(\eta^{x} u_{t x}+\eta^{r} u_{t r}+\eta^{z} u_{t z}+\eta^{t} u_{t t}\right), & \xi^{z}=\eta^{z}
\end{array}
$$

where the arguments of $\eta^{x}, \eta^{r}, \eta^{z}, \eta^{t}$ and second derivatives of $u$ on the righthand sides of (7.25) consist of $x, r, z, t$.

The Legendre transformation of the equation (17.22) results in

$$
\begin{aligned}
& {\left[x\left(q_{z w}-k\right)+u_{r} q_{w w}-e\right] u_{x}+r\left[x q_{z z}+u_{r}\left(q_{z w}-k\right)+c\right]} \\
& +\left(a x+k z+q_{w}\right) u_{z}+t\left(-3 k u_{t}+a u_{r}-q_{z}\right)=2(a P-k u) \\
& +(1 / 6)\left(x^{3} q_{z z z}+3 x^{2} u_{r} q_{z z w}+3 x u_{r}^{2} q_{z w w}+u_{r}^{3} q_{w w w}\right) \\
& +(1 / 2)\left[x^{2} c_{z}-x u_{r}\left(e_{z}-c_{w}\right)-u_{r}^{2} e_{w}\right]+x \rho-u_{r} \sigma+\kappa
\end{aligned}
$$

where $u$ is a solution of the Legendre-transformed second heavenly equation in (5.15),$w=-u_{t}(x, r, z, t)$ should be subsituted in $q, c, e$, their derivatives, $\rho, \sigma$ and $\kappa$ while the transformed potential

$$
P(x, r, z, t)=V\left(x,-u_{r}(x, r, z, t), z,-u_{t}(x, r, z, t)\right)
$$

is determined by the transformed equations (7.21) defining the potential $V$

$$
\begin{aligned}
& u_{t t} P_{x}-u_{t x} P_{t}=2 t\left(u_{r x} u_{t t}-u_{r t} u_{t x}\right)+r\left(u_{t t} u_{x x}-u_{t x}^{2}\right)+2 u_{z} u_{t t} \\
& u_{t t} P_{r}-u_{r t} P_{t}=2 t\left(u_{r r} u_{t t}-u_{r t}^{2}\right)+r\left(u_{r x} u_{t t}-u_{r t} u_{t x}\right)-u_{x} u_{t t}
\end{aligned}
$$


with the only differential compatibility condition coinciding with (5.15).

We need to check if the equation (7.26) can be satisfied for our solutions (6.8). They have again the form (7.10) where

$$
\Phi_{j}=\alpha_{j} t+\beta_{j} r+\gamma_{j} x+\delta_{j} z
$$

$C_{j}$ are arbitrary complex constants and the parameters satisfy (6.9) or (6.17).

Let $n \geq 4$ in (7.10) and $\Phi_{1}, \Phi_{2}, \Phi_{3}, \Phi_{4}$ be linearly independent, i.e. the transformation (7.28) from $t, r, x, z$ to $\Phi_{1}, \Phi_{2}, \Phi_{3}, \Phi_{4}$ is invertible, provided that $\beta_{1} \beta_{2} \beta_{3} \beta_{4} \neq 0$ and the determinant of the matrix of coefficients of (7.28) is non-zero. For the solution (6.8) with the parameters satisfying (6.9) this condition is

$$
\left|\begin{array}{cccc}
\beta_{1} /\left(\gamma_{1}-\beta_{1}\right) & 1 & \gamma_{1} / \beta_{1} & -\gamma_{1}^{2} / \beta_{1}^{2} \\
\beta_{2} /\left(\gamma_{2}-\beta_{2}\right) & 1 & \gamma_{2} / \beta_{2} & -\gamma_{2}^{2} / \beta_{2}^{2} \\
\beta_{3} /\left(\gamma_{3}-\beta_{3}\right) & 1 & \gamma_{3} / \beta_{3} & -\gamma_{3}^{2} / \beta_{3}^{2} \\
\beta_{4} /\left(\gamma_{4}-\beta_{4}\right) & 1 & \gamma_{4} / \beta_{4} & -\gamma_{4}^{2} / \beta_{4}^{2}
\end{array}\right| \neq 0 .
$$

For the solution (6.8) with the parameters satisfying (6.17) the corresponding invertibility condition has the form

$$
\left|\begin{array}{cccc}
\beta_{1} / \gamma_{1} & 1 & \gamma_{1} / \beta_{1} & -\gamma_{1}^{2} / \beta_{1}^{2} \\
\beta_{2} / \gamma_{2} & 1 & \gamma_{2} / \beta_{2} & -\gamma_{2}^{2} / \beta_{2}^{2} \\
\beta_{3} / \gamma_{3} & 1 & \gamma_{3} / \beta_{3} & -\gamma_{3}^{2} / \beta_{3}^{2} \\
\beta_{4} / \gamma_{4} & 1 & \gamma_{4} / \beta_{4} & -\gamma_{4}^{2} / \beta_{4}^{2}
\end{array}\right| \neq 0
$$

Then $t, r, x, z$ can be expressed through $\Phi_{1}, \Phi_{2}, \Phi_{3}, \Phi_{4}$ and the same for $\Phi_{5}, \ldots, \Phi_{n}$, so that $\Phi_{j}$ for $j=1,2,3,4$ can be chosen as new independent variables in (7.26) and the equation (7.26) takes the form (7.13).

In our case solutions of (5.15) are already determined in (6.8) up to arbitrary constants by solving second-order linear equations together with the Legendre-transformed second heavenly equation and hence, having no functional arbitrariness, they cannot satisfy in addition the first-order equation (7.26). Thus for any choice of functions $q, c, e, \rho, \sigma, \kappa$, depending on $z, w=-u_{t}$, arbitrary constants $a, k$ and the potential $P(x, r, z, t)$ satisfying (17.27) the equation (7.26) is not an identity but an equation of the form (7.13). This implies a dependence of the independent variables which is a contradiction that proves nonexistence of the Killing vectors for the metric (5.16) where the potential $u$ is determined by (6.8) with $n \geq 4$ and the conditions (7.29) or (7.30) are satisfied. 
If $n \leq 3$ the above reasoning obviously does not work and Killing vectors may exist.

Thus we have proved the following theorem.

Theorem 3 Metric (5.16) with $u$ defined by (6.8) with the conditions (6.9) or (6.17), $n \geq 4, \beta_{1} \beta_{2} \beta_{3} \beta_{4} \neq 0$ and satisfying the non-degeneracy conditions (7.29) or (7.30) respectively admits no Killing vectors.

\section{Conclusions and discussion}

We have shown that our method of partner symmetries, worked out initially for the elliptic complex Monge-Ampère equation, can be extended to the hyperbolic complex Monge-Ampère equation and the second heavenly equation of Plebañski. This method enables us to construct second order differential constraints which select certain particular sets of solutions invariant with respect to non-local symmetries and hence non-invariant in the usual sense. The advantage of the method is that we deal only with local point symmetries, which build up non-local symmetries, and do not need to work with non-local symmetries explicitly. We found some simple choices of these local partner symmetries for which the Legendre transformation converts the original heavenly equation together with differential constraints to linear equations. We have found their generically non-invariant solutions, dependent on all four variables, and hence new classes of 4-dimensional heavenly metrics without Killing vectors.

The idea of obtaining non-invariant solutions as invariant solutions with respect to non-local symmetry for the hierarchy associated to the second heavenly equation was also suggested by Dunajski and Mason. However, their 'hidden symmetries' constitute a very special class of symmetries which can be generated from local symmetries by repeated applications of the recursion operator and hence have a characteristic property that they can be mapped back to a certain local symmetry by some power of the inverse recursion operator. As a consequence they have at least six differential second-order constraints implied by the hidden symmetries. A class of non-local symmetries constructed from partner symmetries is much more extensive because it consists of symmetries which are linear combinations of local symmetries and those generated from local symmetries by the recursion operators, so that they cannot be mapped to a local symmetry by the action of recursion operators. This additional freedom in symmetries results in wider classes of 
solutions of the heavenly equations since the number of additional differential constraints implied by partner symmetries is typically three, which is less than six in the case of hidden symmetries.

The crucial point of our method is the possibility of linearizing the field equation together with constraints by the Legendre transformation. We have found that this is possible for some simple choices of partner symmetries. An important problem, with which we are occupied, is to work out a criterion which would choose such symmetries for which the linearization is possible.

Another project, that we are working on, is to construct general classes of equations for which our method of partner symmetries could be applied. Characteristic features of such equations should include the divergence structure of the determining equation for symmetries and the condition that a potential for any symmetry should again be a symmetry. We plan to return to this problem soon in a future publication.

\section{Acknowledgements}

One of us, MBS, thanks Thomas Wolf for providing me the latest versions of his Reduce packages Liepde and Applysym for calculating symmetry algebras and performing Legendre transformations. We thank the referees for their criticism and profound remarks that, as we hope, served to a substantial improvement of our paper.

\section{References}

[1] Plebañski J F 1975 J. Math. Phys. 16 2395-2402

[2] Atiyah M F, Hitchin N J and Singer I M 1978 Proc. Roy. Soc. A 362452

[3] Malykh A A, Nutku Y and Sheftel M B 2003 J. Phys. A: Math. Gen. 36 10023-10037

[4] Malykh A A, Nutku Y and Sheftel M B 2003 Class. Quantum Grav. 20 L263-L266

[5] Dunajski M and Mason L J 2003 J. Math. Phys. 44 3430-3454; arXiv:math.DG/0301171 v2 16 Jun 2003. 
[6] Ivanov I T and Roček M 1996 Comm. Math. Phys. 182 291-302

[7] Goldblatt E 1994 Gen. Rel. and Grav. 26979

[8] Aliev A N and Nutku Y 1999 Class. Quantum Gravity 16189

[9] Lechtenfeld O and Popov A D 2000 Int. J. Mod. Phys. A 15 4191-4236

[10] Boyer C P and Winternitz P 1989 J. Math. Phys. 30 1081-1094

[11] Olver P 1986 Applications of Lie Groups to Differential Equations (New York: Springer)

[12] Mason L J and Newman E T 1989 Commun. Math. Phys. 121 659-668

[13] Mason L J and Woodhouse N M J 1996 Integrability, self-duality, and twistor theory (Oxford: Clarendon Press)

[14] Dunajski M and Mason L J 2000 Commun. Math. Phys. 213 641-672

[15] Levi D and Winternitz P 1989 J. Phys. A: Math. Gen. 22 2915-2924 